\title{
Numerical questions in ODE boundary value problems
}

\author{
M. R. Osborne ${ }^{1}$
}

(Received 11 August 2006; revised 02 February 2008)

\begin{abstract}
Classical considerations of stability in ODE initial and boundary problems are mirrored by corresponding properties (stiff stability, distability) in problem discretizations. However, computational categories are not precise, and qualitative descriptors such as 'of moderate size' cannot be avoided where size varies with the sensitivity of the Newton iteration in nonlinear problems, for example. Sensitive Newton iterations require close enough initial estimates. The main tool for providing this in boundary value problems is continuation with respect to a parameter. If stable discretizations are not available, then adaptive meshing is needed to follow rapidly changing solutions. Use of such tools can be necessary in stable nonlinear situations not covered by classical considerations. Implications for the estimation
\end{abstract}

See http://anziamj.austms.org.au/ojs/index.php/ANZIAMJ/article/view/79 for this article, (c) Austral. Mathematical Soc. 2008. Published February 11, 2008. ISSN 1446-8735

${ }^{0}$ This paper is dedicated to Germund Dahlquist, 1925-2005, friend and inspiration. 
problem are sketched. It is shown how to choose appropriate boundary conditions for the embedding method. The simultaneous method is formulated as a constrained optimization problem. It avoids explicit ODE characterization and appears distinctly promising. However, its properties are not yet completely understood.

\section{Contents}

1 Introduction

C900

2 ODE stability

C906

2.1 Initial value problem stability . . . . . . . . . . C906

2.2 Boundary value problem stability . . . . . . . . . . C C908

2.3 Nonlinear stability . . . . . . . . . . . . . . . . . C911

2.4 Stability consequences . . . . . . . . . . . . . C915

$\begin{array}{lll}3 & \text { The estimation problem } & \text { C917 }\end{array}$

3.1 Estimation via embedding . . . . . . . . . . . . . . C C917

3.2 Simultaneous estimation . . . . . . . . . . . . C920

4 In conclusion

C924

References

C924

\section{Introduction}

The stability of the solution of the initial value problem (IVP) for systems of ordinary differential equations has been studied extensively. There is a corresponding theory for numerical schemes which emphasizes the importance of stiff stability in computing solutions of these problems. Stiffly stable formulae permit the evaluation of slowly varying solutions on a grid appropriate 
to the required solution rather than on one capable of accurately following the most rapidly decaying solutions supported by the differential equation. However, computational problems can have requirements to produce numerical results for problems with solutions which involve both growth and decay and so may require fine grids and adaptive meshing procedures. Boundary value problems (BVP) involve a global statement which makes corresponding results more elusive, but problems which are similar in kind occur. The analogous property here is called di-stability. This permits the required solution to be evaluated on an appropriate grid rather than one determined by the most rapidly varying solutions The estimation problem is an inverse problem somewhat akin to the BVP. It arises in trying to quantify system properties using information obtained by observing solution trajectories, typically in the presence of noise. Important in applications, the requirement here is to clarify the role of intrinsic properties of the differential equation in the well-determinedness or otherwise of the estimation problem solution.

The differential equation is written

$$
\frac{d \mathbf{x}}{d t}=\mathbf{f}(t, \mathbf{x})
$$

where $\mathbf{x} \in \mathbb{R}^{m}, \mathbf{f} \in \mathbb{R}^{1} \times \mathbb{R}^{m} \rightarrow \mathbb{R}^{m}$, and the forcing function $\mathbf{f}$ is assumed to have any required degree of smoothness. Boundary conditions have the form

$$
B_{0} \mathbf{x}(0)+B_{1} \mathbf{x}(1)=\mathbf{b},
$$

where $\mathbf{b} \in \mathbb{R}^{m}$ and $B_{0}, B_{1} \in \mathbb{R}^{m} \rightarrow \mathbb{R}^{m}$. Rank conditions on $B_{0}$ and $B_{1}$ are necessary for the existence of a unique solution (see (6)). Boundary conditions are separated if no row of (2) couples solution values at both boundary points. This formulation contains the IVP as the special separated case $B_{0}=I$ and $B_{1}=0$. The IVP is distinguished because relatively weak conditions are sufficient to guarantee a local solution. The general boundary formulation also suffices for multipoint problems which can be reduced to BVP form by mapping each of the subintervals into $[0,1]$. 
Conditions for the existence of solutions of the BVP are obtained by first considering the linear differential equation

$$
\frac{d \mathbf{x}}{d t}=A(t) \mathbf{x}+\mathbf{q}(t) .
$$

Associated with this equation are IVPs for the fundamental matrix $X(t, \xi)$ satisfying

$$
\frac{d X}{d t}=A(t) X, \quad X(\xi, \xi)=I,
$$

and the particular integral $\mathbf{w}(t, \xi)$ satisfying

$$
\frac{d \mathbf{w}}{d t}=A(t) \mathbf{w}+\mathbf{q}(t), \quad \mathbf{w}(\xi, \xi)=0 .
$$

The solution of the BVP is now written by supposition

$$
\mathbf{x}=X(t, 0) \mathbf{x}(0)+\mathbf{w}(t, 0),
$$

where $\mathbf{x}(0)$ must be chosen to satisfy the boundary value equations

$$
\left(B_{0}+B_{1} X(1,0)\right) \mathbf{x}(0)=\mathbf{b}-B_{1} \mathbf{w}(1,0) .
$$

This is satisfied provided $B_{0}+B_{1} X(1,0)$ has a bounded inverse. Solution conditions in the nonlinear case are obtained by linearizing around an assumed solution and applying the Newton-Kantorovich theory [6].

Knowledge of the fundamental matrix permits explicit solution representations to be written down in the linear case. In particular, the Green's matrix is

$$
\begin{aligned}
G(t, s) & =X(t)\left[B_{0} X(0)+B_{1} X(1)\right]^{-1} B_{0} X(0) X^{-1}(s), \quad t>s, \\
& =-X(t)\left[B_{0} X(0)+B_{1} X(1)\right]^{-1} B_{1} X(1) X^{-1}(s), \quad t<s .
\end{aligned}
$$

Note that $G$ is independent of the form of initial condition on the fundamental matrix provided only that the result has full rank. The size of the Green's 
matrix governs the sensitivity of the BVP solution to perturbations in $\mathbf{q}(t)$. This is clearly important in stability considerations. The quantity

$$
\alpha=\max _{0 \leq t, s \leq 1}\|G(t, s)\|_{2}
$$

is called the stability constant.

An important related problem is the estimation problem. Here the argument of the forcing function $\mathbf{f}$ in (1) contains also a vector of parameters $\boldsymbol{\beta} \in \mathbb{R}^{p}$,

$$
\mathbf{f} \leftarrow \mathbf{f}(t, \mathbf{x}, \boldsymbol{\beta}) .
$$

This vector of parameters is to be estimated using information gained by observing a solution trajectory in the presence of noise at a set of points $T_{n}=\left\{t_{1}, t_{2}, \ldots, t_{n}\right\}$. This information is assumed to have the form

$$
\mathbf{y}_{i}=H \mathbf{x}\left(t_{i}, \boldsymbol{\beta}\right)+\varepsilon_{i}, \quad t_{i} \in T_{n},
$$

where $\mathbf{y}_{i} \in \mathbb{R}^{k}, k \leq m, H: \mathbb{R}^{m} \rightarrow \mathbb{R}^{k}$ has rank $k$, and $\boldsymbol{\varepsilon}_{i} \sim N\left(0, \sigma^{2} I\right)$ are independent samples from a random process. Differences with the BVP arise not only from the presence of the noise process, but also from a requirement that a sufficiently rich set of observations be available. A minimum condition assumed is that $n k>m$ so the problem is formally strictly over-determined. This means that the best that can be done in general is to seek a solution that minimizes a goodness of fit criterion with respect to the observed data. Here this criterion is assumed to be

$$
F(\boldsymbol{\beta})=\sum_{i=1}^{n}\left\|\mathbf{y}_{i}-H \mathbf{x}\left(t_{i}, \boldsymbol{\beta}\right)\right\|_{2}^{2} .
$$

It can be interpreted either in a least squares or maximum likelihood sense. The resulting optimization problem has two forms depending on the manner of generating the comparison functions $\mathbf{x}(t, \boldsymbol{\beta})$.

Embedding Here boundary matrices $B_{0}$ and $B_{1}$ are selected in order to embed the comparison function in a family of BVPs. Now the appropriate 
right hand side vector $\mathbf{b}$ becomes a vector of auxiliary parameters to be determined as part of the estimation problem. The selection of $B_{0}$ and $B_{1}$ has to be specified a priori in a manner compatible with stability constraints on the problem, and this approach has the further disadvantage that a (in general nonlinear) BVP must be solved for each set of function values required in (9) at each Newton iteration. However, it does separate the estimation and solution components of the problem making it relatively easy to draw on existing software.

Simultaneous The idea is to impose a discretized form of the differential equation as a set of equality constraints on the problem of minimizing $F$. Estimates of $\boldsymbol{\beta}$ and the state variables $\mathbf{x}\left(t_{i}, \boldsymbol{\beta}\right)$ are then generated simultaneously by solving the resulting constrained optimization problem. Here it simplifies discussion to combine the state vector with the parameter vector $\mathbf{x}^{T} \leftarrow\left[\begin{array}{ll}\mathbf{x}^{T} & \boldsymbol{\beta}^{T}\end{array}\right]$ and to augment the differential equation system with the additional equations $d \boldsymbol{\beta} / d t=0$. The simultaneous method is potentially more computationally efficient. However, BVP implementation questions such as the selection of an appropriately graded mesh become more complicated because the estimation and solution components are now more closely coupled.

An important aspect of the estimation problem is the selection of the points $t_{i} \in T_{n}$. It is necessary to distinguish two classes of experiment.

1. One in which observations are not available outside a finite interval which is assumed to be $[0,1]$. This is the situation considered here. Full information requires that $T_{n}$ can be generated for arbitrarily large $n$. These experiments are called planned if the sets $T_{n}$ satisfy the condition

$$
\frac{1}{n} \sum_{i=1}^{n} v\left(t_{i}\right) \rightarrow \int_{0}^{1} v(t) d \rho(t) \quad \text { as } \quad n \rightarrow \infty,
$$

for all $v(t) \in C[0,1]$. This requirement just reflects the requirement that the non-negative density function $\rho$ is associated with the mecha- 
nism which must be set in place to generate an unbounded number of observations.

2. The alternative situation is one in which observations on a trajectory for arbitrarily large time contain parametric information. An important class of these problems is the class of stationary processes for which a rather different and distinctly sophisticated body of theory has been developed. One problem of this type which can be posed as an estimation problem is that of determining frequencies. These are functions of the coefficients in a linear ODE with constant coefficients.

The stochastic aspects of the estimation problem has an important bearing on the choice of $T_{n}$.

- The asymptotic analysis of the effects of noisy data on maximum likelihood estimates of the parameters shows that this gets small no faster than $O\left(n^{-1 / 2}\right)$ under planned experiment conditions. A higher rate $\left(O\left(n^{-3 / 2}\right)\right)$ is theoretically possible in maximum likelihood estimates in the frequency estimation problem, but direct maximization is not the way to obtain these quantities [12].

- It is not difficult to obtain ODE discretizations that give errors at most $O\left(n^{-2}\right)$.

This suggests that the trapezoidal rule provides an adequate discretization. It has the form

$$
\mathbf{c}_{i}\left(\mathbf{x}_{c}\right)=\mathbf{x}_{i+1}-\mathbf{x}_{i}-\frac{h}{2}\left(\mathbf{f}_{i+1}+\mathbf{f}_{i}\right)=0, \quad i=1,2, \ldots, n-1,
$$

with $\mathbf{x}_{i}=\mathbf{x}\left(t_{i}, \boldsymbol{\beta}\right), \mathbf{x}_{c}$ the composite vector with sub-vector components $\mathbf{x}_{i}$, and $h$ the discretization mesh spacing. It is known to be endowed with attractive properties [4]. 
A loose thread to link the stability topics and the embedding problem is provided by several examples involving the Mattheij equation [1]. This equation possesses a strong dichotomy which is the BVP analogue of IVP stability. Numerical results illustrate the importance of the property of di-stability which extends to the BVP context the requirement that the discretization method be stiffly stable.

\section{ODE stability}

\subsection{Initial value problem stability}

Consider first the stability of the IVP

$$
\frac{d \mathbf{x}}{d t}=\mathbf{f}(t, \mathbf{x}), \quad \mathbf{x}(0)=\mathbf{b} .
$$

Here, initial value problem stability (IVs) means that different solutions with close initial conditions, $\mathbf{x}_{1}(0)$ and $\mathbf{x}_{2}(0)$, remain close in an appropriate sense.

- Let $\left\|\mathbf{x}_{1}(t)-\mathbf{x}_{2}(t)\right\|$ remain bounded $(\rightarrow 0)$ as $t \rightarrow \infty$. This corresponds to weak (strong) IVs. Note that systems with bounded oscillatory solutions may well be weakly stable but the classification may not be particularly useful.

- Computation introduces the idea of stiff discretizations which preserve the stability characteristics of (11) in the sense that decaying solutions of the differential equation are mapped onto decaying solutions of the computational problem. The advantage is that the computation does not have to follow rapidly decaying solutions in detail. This is one area where there are genuine nonlinear results - for example, Butcher's work on BN-stability of Runge-Kutta methods [2]. 
However, not all relevant IVPs are stable. The classical BVP solution method of multiple shooting provides an example [10]. This requires computation of the multiple shooting matrix of the linearized equation:

$$
\left[\begin{array}{ccccc}
-X\left(t_{2}, t_{1}\right) & I & & & \\
& -X\left(t_{3}, t_{2}\right) & I & & \\
& & \ddots & \ddots & \\
B_{0} & & & & B_{1}
\end{array}\right] .
$$

The problem is that the IVP for computing $X\left(t_{i+1}, t_{i}\right)$ could well be unstable in both forward and backward directions when the BVP has a well determined solution. This does not mean progress cannot be made. This is a consequence of Dahlquist's famous theorem [3], "consistency plus stability implies convergence as $h \rightarrow 0$ ", which does not require IVP stability. However, its setting implies exact arithmetic. The problem for practical computation is a form of numerical instability. This occurs in trying to follow a decreasing solution in the presence of rapidly increasing solutions. Rounding error introduces components of the fast solutions, and they will eventually swamp the one required. Compromise is necessary. Here this takes the form of restrictions on the length of the interval of integration. This control in multiple shooting is provided by the choice of the $\left\{t_{i}\right\}$. Multiple shooting in this form appears to require accurate computation of all solutions, and this is potentially a weakness.

This discussion is readily illustrated in the constant coefficient case. Consider the ODE

$$
\mathbf{f}(t, \mathbf{x})=A \mathbf{x}-\mathbf{q} .
$$

If $A$ is non-defective then weak IVS requires the eigenvalues $\lambda_{i}(A)$ to satisfy $\Re\left\{\lambda_{i}\right\} \leq 0$ while this inequality must be strict for strong IVs. A one step discretization of the ODE (ignoring the $\mathbf{q}$ contribution) is written

$$
\mathbf{x}_{i+1}=T_{h}(A) \mathbf{x}_{i},
$$


where $T_{h}(A)$ is the amplification matrix. A stiff discretization requires the stability inequalities to map into the condition $\left|\lambda_{i}\left(T_{h}\right)\right| \leq 1$. For the trapezoidal rule

$$
\begin{aligned}
\left|\lambda_{i}\left(T_{h}\right)\right| & =\left|\frac{1+h \lambda_{i}(A) / 2}{1-h \lambda_{i}(A) / 2}\right|, \\
& \leq 1 \quad \text { if } \Re\left\{\lambda_{i}(A)\right\} \leq 0 .
\end{aligned}
$$

\subsection{Boundary value problem stability}

The generalization of IVS that is appropriate for linear differential equations is provided by the property of dichotomy: de Hoog and Mattheij [5] wrote the key article discussing the computational context. Only a weak form of dichotomy is considered here. It requires that there exists a projection $P$ depending on the choice of $X$ such that, given the complementary solution sets

$$
S_{1} \leftarrow\left\{X P \mathbf{w}, \mathbf{w} \in \mathbb{R}^{m}\right\}, \quad S_{2} \leftarrow\left\{X(I-P) \mathbf{w}, \mathbf{w} \in \mathbb{R}^{m}\right\},
$$

then for all $s, t$

$$
\begin{aligned}
& \phi \in S_{1} \Rightarrow \frac{\|\phi(t)\|_{\infty}}{\|\phi(s)\|_{\infty}} \leq \kappa, \quad t \geq s, \\
& \phi \in S_{2} \Rightarrow \frac{\|\phi(t)\|_{\infty}}{\|\phi(s)\|_{\infty}} \leq \kappa, \quad t \leq s .
\end{aligned}
$$

This is the structural property that connects linear BVP stability with the detailed behaviour of the range of possible solutions. However, the BVP is specified on a finite interval. This means that there is always a bounded $\kappa$ on that interval provided the fundamental matrix is bounded, . The additional feature in the computational context is that a modest $\kappa$ is required for $t, s \in$ $[0,1]$. The key result is that if $X$ satisfies $B_{0} X(0)+B_{1} X(1)=I$, then $P=B_{0} X(0)$ is a suitable projection in sense that for separated boundary 
conditions the choice $\kappa=\alpha$ is allowed where $\alpha$ is the stability constant. There is an intimate connection between stability and dichotomy. Dichotomy permits a form of generalization of A-stability to the BVP case.

- The dichotomy projection separates increasing and decreasing solutions of the differential equation. Compatible boundary conditions pin down rapidly decreasing solutions at 0 , and rapidly increasing solutions at 1 .

- The discretization needs similar property in order that the given boundary conditions exercise the same control on the discretized system.

- This requires solutions of the ODE which are rapidly increasing (decreasing) be mapped into solutions of the discretization which are increasing (decreasing) in magnitude.

This property is called di-stability in [7]. They show that the trapezoidal rule is di-stable in the constant coefficient case. The argument is straightforward:

$$
\lambda(A)>0 \Rightarrow\left|\frac{1+h \lambda(A) / 2}{1-h \lambda(A) / 2}\right|>1 .
$$

Example 1 Mattheij suggested a problem which provides an interesting test of discretization methods. Consider the differential system defined by

$$
\begin{aligned}
A(t) & =\left[\begin{array}{ccc}
1-19 \cos 2 t & 0 & 1+19 \sin 2 t \\
0 & 19 & 0 \\
-1+19 \sin 2 t & 0 & 1+19 \cos 2 t
\end{array}\right], \\
\mathbf{q}(t)= & {\left[\begin{array}{c}
e^{t}(-1+19(\cos 2 t-\sin 2 t)) \\
-18 e^{t} \\
e^{t}(1-19(\cos 2 t+\sin 2 t))
\end{array}\right] . }
\end{aligned}
$$


TABLE 1: Boundary point values - stable. computation

\begin{tabular}{c|ccc|ccc} 
& \multicolumn{3}{|c|}{$h=0.1$} & \multicolumn{3}{c}{$h=.01$} \\
\hline $\mathbf{x}(0)$ & 1.0000 & .9999 & .9999 & 1.0000 & 1.0000 & 1.0000 \\
$\mathbf{x}(1)$ & 2.7183 & 2.7183 & 2.7183 & 2.7183 & 2.7183 & 2.7183
\end{tabular}

Here the right hand side is chosen so that slowly varying $\mathbf{z}(t)=e^{t} \mathbf{e}$ satisfies the differential equation. The fundamental matrix displays two fast solutions and one slow solution showing that this system exhibits strong dichotomy:

$$
X(t, 0)=\left[\begin{array}{ccc}
e^{-18 t} \cos t & 0 & e^{20 t} \sin t \\
0 & e^{19 t} & 0 \\
-e^{-18 t} \sin t & 0 & e^{20 t} \cos t
\end{array}\right] .
$$

For boundary data with two terminal conditions, one initial condition, and right-hand side chosen to match the exponential solution:

$$
B_{0}=\left[\begin{array}{ccc}
0 & 0 & 0 \\
0 & 0 & 0 \\
1 & 0 & 0
\end{array}\right], \quad B_{1}=\left[\begin{array}{ccc}
1 & 0 & 0 \\
0 & 1 & 0 \\
0 & 0 & 0
\end{array}\right], \quad \mathbf{b}=\left[\begin{array}{c}
e \\
e \\
1
\end{array}\right],
$$

the trapezoidal rule discretization scheme gives the results in Table 1. These computations are apparently satisfactory.

In contrast, posing two initial and one terminal condition:

$$
B_{0}=\left[\begin{array}{lll}
0 & 0 & 1 \\
0 & 0 & 0 \\
1 & 0 & 0
\end{array}\right], \quad B_{1}=\left[\begin{array}{lll}
0 & 0 & 0 \\
0 & 1 & 0 \\
0 & 0 & 0
\end{array}\right], \quad \mathbf{b}=\left[\begin{array}{l}
1 \\
e \\
1
\end{array}\right]
$$

gives the results in Table 2 The effects of instability are seen clearly in the first and third solution components.

The feature of this example is the role of di-stability. Consider the trapezoidal rule denominator in (12). This suggests large and spurious amplification is likely in case $h=0.1$; but this is not evident in the stable computation. 
TABLE 2: Boundary point values - unstable computation.

\begin{tabular}{c|ccc|ccc} 
& \multicolumn{3}{|c|}{$h=0.1$} & \multicolumn{3}{c}{$h=.01$} \\
\hline $\mathbf{x}(0)$ & 1.0000 & .9999 & 1.0000 & 1.0000 & 1.0000 & 1.0000 \\
$\mathbf{x}(1)$ & $-7.9+11$ & 2.7183 & $-4.7+11$ & $2.03+2$ & 2.7183 & $1.31+2$
\end{tabular}

However, the unstable case corresponding to $h=0.1$ does show more influence of instability than the case $h=.01$. The small denominator in (12) suggests the likely explanation.

\subsection{Nonlinear stability}

Stability in nonlinear problems becomes a property of the linear problem governing the behaviour of perturbations about a current trajectory. In this sense it is a local property. Easy nonlinear problems are associated with relatively slow perturbation growth. Such problems can be expected to have the property that Newton's method applied to solve the discretized problem will have a reasonable domain of convergence. The linear IVP/BVP stability requirements are inflexible in the sense that solutions must not depart too far from the classification as increasing/decreasing. Important conflicting examples occur in the linearized equations associated with dynamical system trajectories. These include solution trajectories which

- can have a stable character-for example, limiting trajectories which attract neighbouring orbits;

- and have linearized systems which switch between increasing and decreasing modes in a manner characteristic of oscillatory behaviour. If this switch is rapid then it will be difficult to satisfy the dichotomy partitioning inequalities with a modest bound. This is likely to make more 
difficult the solution of the nonlinear problem by Newton's method.

Limit cycle behavior provides a familiar example that is of this type. Intriguingly it shares some of the properties of stationary processes in the sense that observations contain trajectory information for all $t$.

Example 2 To exhibit limit cycle behaviour consider the FitzHugh-Nagumo equations:

$$
\begin{aligned}
& \frac{d V}{d t}=\gamma\left(V-\frac{V^{3}}{3}+R\right), \\
& \frac{d R}{d t}=-\frac{1}{\gamma}(V-\alpha-\beta R) .
\end{aligned}
$$

Solution components for $\alpha=.2, \beta=.2, \gamma=1$ are illustrated in Figure 1. Note that the positive and negative components of the individual cycles are not exact opposites.

Example 3 The Van der Pol equation is

$$
\frac{d^{2} x}{d t^{2}}-\lambda\left(1-x^{2}\right) \frac{d x}{d t}+x=0 .
$$

This provides a difficult ODE example with difficulty increasing with $\lambda$. The solutions here are exactly periodic. Stability is illustrated by convergence of trajectories from nearby initial points to the limit cycle. Figure 2 illustrates rapid convergence to the limit cycle for $\lambda=1,10$ computed using standard Scilab code. Computational problems occur because of the need to follow rapidly changing trajectories in detail. The clustering of integration points near the approximately vertical section of each trajectory shows the importance of adaptive mesh selection. Note the rapid convergence to the limiting trajectory showing this is certainly a stable situation. 

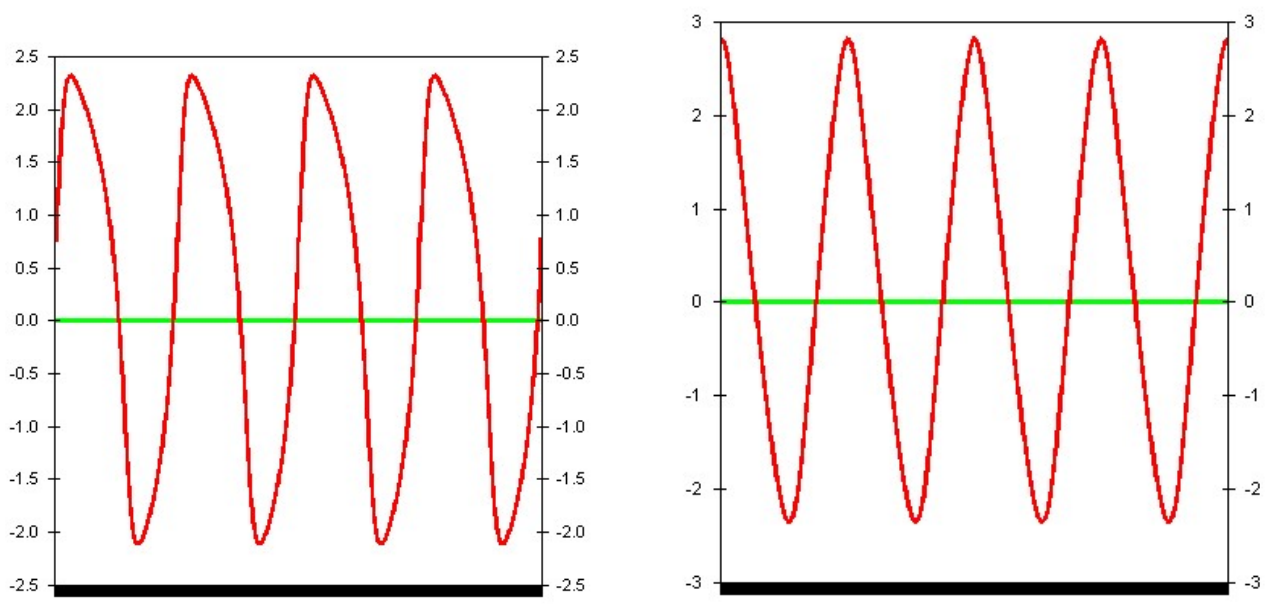

Figure 1: FitzHugh-Nagumo BVP solutions $V$ and $R$.

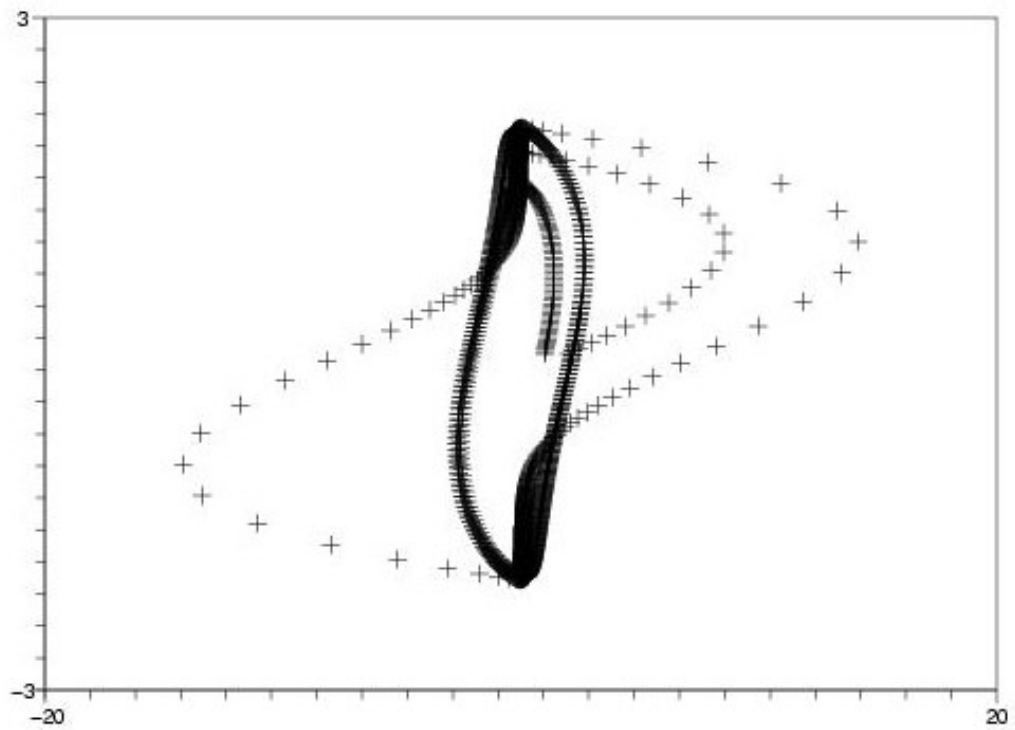

Figure 2: Scilab plot of Van der Pol trajectories for $\lambda=1,10$. 


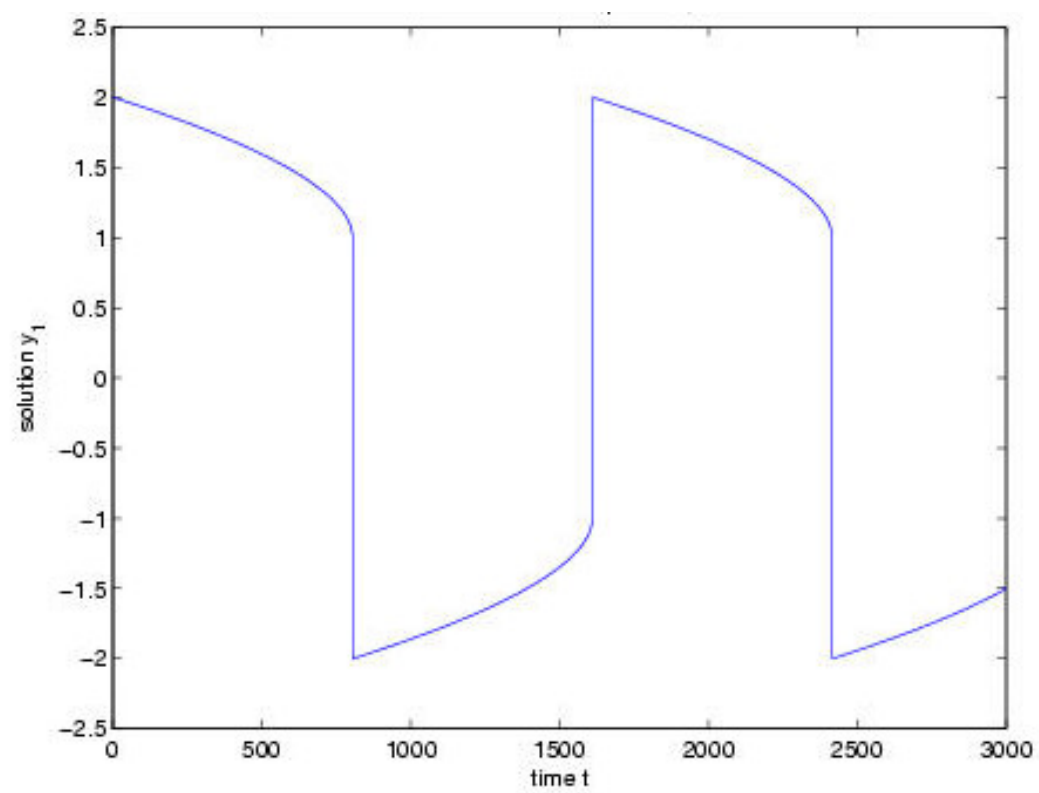

Figure 3: Matlab plot of state variable for $\lambda=1000, x_{1}(0)=2$.

Matlab also uses this example in demonstration software but the output is less useful as it gives state information but not the derivative values for the case $\lambda=1000$ (Figure 3). This plot of a difficult case implies an excellent IVP solver. The starting values $(2,0)$ used are rather special as

$$
x_{1}(0)=2+\frac{1}{3} \alpha \lambda^{-4 / 3}-\frac{16}{27} \lambda^{-2} \ln (\lambda)+O\left(\lambda^{-2}\right),
$$

where $\alpha=2.33811 \cdots$.

Example 4 The Van der Pol equation is exactly cyclic so the problem of computing a half cycle is cast in BVP form on the interval $[0,2]$ by making the transformation $s=4 t / T$. The unknown interval length is treated as an 
additional variable by setting $x_{3}=T / 4$. The resulting ODE system becomes

$$
\begin{aligned}
& \frac{d x_{1}}{d s}=x_{2}, \\
& \frac{d x_{2}}{d s}=\lambda\left(1-x_{1}^{2}\right) x_{2} x_{3}-x_{1} x_{3}^{2}, \\
& \frac{d x_{3}}{d s}=0 .
\end{aligned}
$$

The appropriate boundary data are

$$
B_{0}=\left[\begin{array}{lll}
0 & 1 & 0 \\
0 & 0 & 0 \\
1 & 0 & 0
\end{array}\right], \quad B_{1}=\left[\begin{array}{ccc}
0 & 0 & 0 \\
0 & 1 & 0 \\
1 & 0 & 0
\end{array}\right], \quad \mathbf{b}=0
$$

The nonlinear system has the trivial solution $\mathbf{x}=0$ so it is necessary to choose appropriate nonzero initial estimates. Here this has been done by taking the periodic solution for $\lambda=0$ with $x_{1}(0)=2$ and $x_{2}(0)=0$ as the initial estimate. This gives convergence for the Newton iteration for $\lambda=1$ and continues to work for $\lambda \leq 5$. Continuation with $\Delta \lambda=1$ is used for higher values. The fixed discretizations exemplified are $h=.01$ and $h=.001$. This is not ideal for this problem as the IVP computations have illustrated the importance of adaptive meshing. The BVP results for $\lambda=10, h=.001$ are given in Figure 4. These reinforce the need for the use of appropriately graded mesh selection.

\subsection{Stability consequences}

The ODE stability conditions provide sharp distinctions - in part because they are specifying global properties. Computational requirements force compromise. In the IVP this is provided by various control devices: for example, automatic step length control. There are two classes of computational stability problem: 

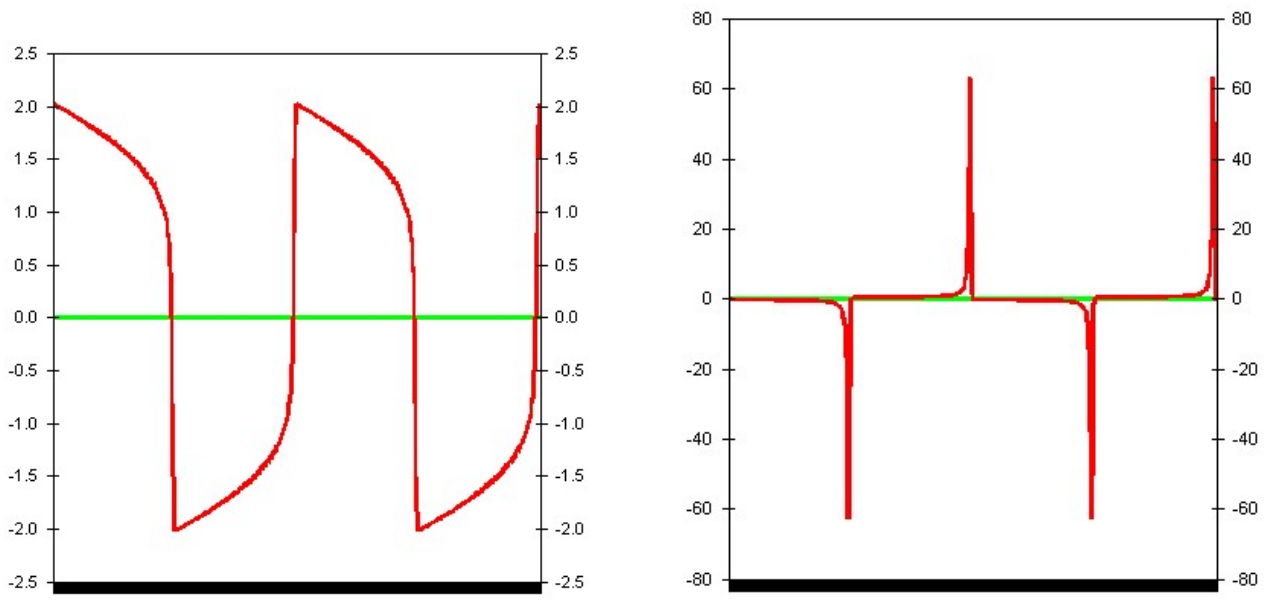

Figure 4: Van der Pol solution $x_{1}$ and $x_{2}$ for $\lambda=10$.

- The Dahlquist root condition [3] is not satisfied. In this case errors grow with $n$ and so are unbounded as $h \rightarrow 0$. This occurs whether or not the original problem is unstable.

- In unstable IVPs a computed slow solution will be swamped eventually as a result of the growth of rounding error induced perturbations which in a worst case grow like the Gronwall Lemma bound $\gamma \exp (K t)$. This is the problem which multiple shooting seeks to control. This device appears not be necessary in stable BVPs if di-stable discretizations are used.

In the BVP stability discussion the dichotomy considerations are restricted to a finite interval on which we ask for 'moderate' $\kappa$. Here the individual terms in the inverse of the multiple shooting matrix can be interpreted using the Green's matrix. If $\kappa$ is large then the BVP will be associated with a sensitive Newton iteration because the inverse Jacobian matrices must contain terms of size $O(\kappa)$. Available tools for overcoming this problem include: 
- use of adaptive mesh control in positioning discretization points - but this may be difficult if good initial estimates are not available;

- adaptive continuation with respect to a parameter in order to move from a known to a required BVP solution in a sequence of steps in which the current solution provides a good enough initial estimate for convergence of the Newton iteration at the next continuation increment.

\section{The estimation problem}

\subsection{Estimation via embedding}

The embedding form of the estimation problem sketched in the introduction leads to a nonlinear least squares problem to minimize (9) for the unknown parameters $\boldsymbol{\beta}$ and $\mathbf{b}$. This can be solved by an application of the GaussNewton method [9] once the form of the boundary conditions needed to specify the embedding have been set. This will be done by noting that a good choice should lead to a relatively well-conditioned linear system in setting up the linear least squares problem for the Gauss-Newton correction. To see what is involved note that the trapezoidal rule discretization of the differential equation (10) has the form

$$
0=\mathbf{c}_{i}\left(\mathbf{x}_{i}, \mathbf{x}_{i+1}\right)=\mathbf{c}_{i i}\left(\mathbf{x}_{i}\right)+\mathbf{c}_{i(i+1)}\left(\mathbf{x}_{i+1}\right), \quad i=1,2, \ldots, n-1 .
$$

Here the state variable contributions enter additively. As a consequence the gradient system has the block bi-diagonal matrix

$$
C=\left[\begin{array}{ccccc}
C_{11} & C_{12} & & & \\
& C_{22} & C_{23} & & \\
& & & \ddots & \\
& & & \ddots & C_{(n-1) n}
\end{array}\right],
$$


where $C_{i j}=\nabla_{\mathbf{x}_{j}} \mathbf{c}_{i}$. Consider the orthogonal $Q$ times upper triangular $U$ factorization of this system with the first column permuted to the last place:

$$
\left[\begin{array}{llll|l}
C_{12} & & & & C_{11} \\
C_{22} & C_{23} & & & \\
& & & & \\
\hline & & C_{(n-1)(n-1)} & C_{(n-1) n} & 0
\end{array}\right] \rightarrow Q\left[\begin{array}{ccc|c} 
& U & Z \\
\hline 0 & \cdots & D & G
\end{array}\right] .
$$

This step is independent of the boundary conditions. It permits a solution representation for linear systems of the form

$$
\mathbf{x}_{i}=V_{i} \mathbf{x}_{1}+W_{i} \mathbf{x}_{n}+\mathbf{w}_{i}, \quad i=2, \ldots, n-1 .
$$

with unknowns $\mathbf{x}_{1}$ and $\mathbf{x}_{n}$. Factorization by orthogonal cyclic reduction associates $\left\{V_{i}, W_{i}, \mathbf{w}_{i}\right\}$ with solutions of the ODE system of twice the order [11]:

$$
\left\{\frac{d}{d t}+A^{T}(t)\right\}\left\{\frac{d}{d t}-A(t)\right\} \mathbf{z}=\cdots,
$$

where $A$ is the coefficient matrix of the linear ODE. Does a dichotomy result for this system follow from dichotomy for the original? The result is true for systems with constant coefficients. The orthogonal factorization suggested here is not the same as cyclic reduction, but the performance appears similar.

If boundary conditions are inserted at this point then there results a system for $\mathbf{x}_{1}$ and $\mathbf{x}_{n}$ with matrix $\left[\begin{array}{cc}D & G \\ B_{1} & B_{0}\end{array}\right]$. Orthogonal $S$ times lower triangular $L$ factorization provides a useful strategy.

$$
\left[\begin{array}{ll}
D & G
\end{array}\right]=\left[\begin{array}{ll}
L & 0
\end{array}\right]\left[\begin{array}{l}
S_{1}^{T} \\
S_{2}^{T}
\end{array}\right]
$$

It follows that the system determining $\mathbf{x}_{1}$ and $\mathbf{x}_{n}$ is best conditioned by choosing

$$
\left[\begin{array}{ll}
B_{1} & B_{0}
\end{array}\right]=S_{2}^{T} \text {. }
$$


This choice of $B_{0}$ and $B_{1}$ depends only on the ODE, but it does depend on $\boldsymbol{\beta}$ in the estimation problem as a consequence. Its use is illustrated in Example 5.

To set up the Gauss-Newton iteration let $\nabla_{(\beta, b)} \mathbf{x}=\left[\begin{array}{ll}\partial \mathbf{x} / \partial \boldsymbol{\beta} & \partial \mathbf{x} / \partial \mathbf{b}\end{array}\right]$, $\mathbf{r}_{i}=\mathbf{y}_{i}-H \mathbf{x}\left(t_{i}, \boldsymbol{\beta}, \mathbf{b}\right)$. Then the gradient of $F$ is

$$
\nabla_{(\beta, b)} F=-2 \sum_{i=1}^{n} \mathbf{r}_{i}^{T} H \nabla_{(\beta, b)} \mathbf{x}_{i} .
$$

The gradient terms with respect to $\boldsymbol{\beta}$ are found by solving the BVPs

$$
\begin{gathered}
B_{0} \frac{\partial \mathbf{x}}{\partial \boldsymbol{\beta}}(0)+B_{1} \frac{\partial \mathbf{x}}{\partial \boldsymbol{\beta}}(1)=0, \\
\frac{d}{d t} \frac{\partial \mathbf{x}}{\partial \boldsymbol{\beta}}=\nabla_{x} \mathbf{f} \frac{\partial \mathbf{x}}{\partial \boldsymbol{\beta}}+\nabla_{\beta} \mathbf{f},
\end{gathered}
$$

while the corresponding terms with respect to $\mathbf{b}$ satisfy the BVPS

$$
\begin{gathered}
B_{0} \frac{\partial \mathbf{x}}{\partial \mathbf{b}}(0)+B_{1} \frac{\partial \mathbf{x}}{\partial \mathbf{b}}(1)=I, \\
\frac{d}{d t} \frac{\partial \mathbf{x}}{\partial \mathbf{b}}=\nabla_{x} \mathbf{f} \frac{\partial \mathbf{x}}{\partial \mathbf{b}} .
\end{gathered}
$$

Example 5 Consider the modification of the Mattheij problem with parameters $\beta_{1}^{*}=\gamma$ and $\beta_{2}^{*}=2$ corresponding to the solution $\mathbf{x}\left(t, \boldsymbol{\beta}^{*}\right)=e^{t} \mathbf{e}$ :

$$
\begin{aligned}
A(t) & =\left[\begin{array}{ccc}
1-\beta_{1} \cos \beta_{2} t & 0 & 1+\beta_{1} \sin \beta_{2} t \\
0 & \beta_{1} & 0 \\
-1+\beta_{1} \sin \beta_{2} t & 0 & 1+\beta_{1} \cos \beta_{2} t
\end{array}\right], \\
\mathbf{q}(t)= & {\left[\begin{array}{c}
e^{t}(-1+\gamma(\cos 2 t-\sin 2 t)) \\
-(\gamma-1) e^{t} \\
e^{t}(1-\gamma(\cos 2 t+\sin 2 t))
\end{array}\right] . }
\end{aligned}
$$


TABLE 3: Embedding method: Gauss-Newton results for the Mattheij problem

$$
\begin{array}{cc}
H=\left[\begin{array}{lll}
1 / 3 & 1 / 3 & 1 / 3
\end{array}\right] & H=\left[\begin{array}{ccc}
.5 & 0 & .5 \\
0 & 1 & 0
\end{array}\right] \\
h=.02, \gamma=10, \sigma=.1 & h=.02, \gamma=10, \sigma=.1 \\
14 \text { iterations } & 5 \text { iterations } \\
h=.02, \gamma=20, \sigma=.1 & h=.02, \gamma=20, \sigma=.1 \\
11 \text { iterations } & 9 \text { iterations } \\
h=.004, \gamma=10, \sigma=.1 & h=.004, \gamma=10, \sigma=.1 \\
9 \text { iterations } & 4 \text { iterations } \\
h=.004, \gamma=20, \sigma=.1 & h=.004, \gamma=20, \sigma=.1 \\
8 \text { iterations } & 5 \text { iterations }
\end{array}
$$

In the numerical experiments optimal boundary conditions are set at the first iteration. The aim is to recover estimates of $\boldsymbol{\beta}^{*}$ and $\mathbf{b}^{*}$ from simulated data $e^{t_{i}} H \mathbf{e}+\boldsymbol{\varepsilon}_{i}, \boldsymbol{\varepsilon}_{i} \sim N(0, .01 I)$ using Gauss-Newton, stopping when $|\nabla F \mathbf{z}|<$ $10^{-8}$ where $\mathbf{z}$ is the predicted correction. Results are given in Table 3.

Here the effect of varying $\boldsymbol{\beta}$ and $\mathbf{b}$ proves negligible. The angle between the initial conditions and the optimal conditions for the subsequent values of $\boldsymbol{\beta}$ and $\mathbf{b}$ are determined by $\left\|\left[\begin{array}{ll}B_{1} & B_{2}\end{array}\right]_{1}\left[\begin{array}{ll}B_{1} & B_{2}\end{array}\right]_{k}^{T}-I\right\|_{F}<10^{-3}, k>1$. This example possesses a dichotomy so these results confirm the efficacy of the embedding method for stable problems.

\subsection{Simultaneous estimation}

The simultaneous method outlined in the introduction leads to the optimization problem:

$$
\min _{\mathbf{x}_{c}} F\left(\mathbf{x}_{c}\right) ; \quad \mathbf{c}_{i}\left(\mathbf{x}_{c}\right)=0, \quad i=1,2, \ldots, n-1,
$$


where $\mathbf{x}_{c} \in \mathbb{R}^{n m}$ is the composite vector with block sub-vectors $\mathbf{x}_{1}, \mathbf{x}_{2}, \ldots, \mathbf{x}_{n}$, and where the individual state and parameter vectors are bundled together to form composite state sub-vectors. Introducing the Lagrangian function

$$
\mathcal{L}=F\left(\mathbf{x}_{c}\right)+\sum_{i=1}^{n-1} \boldsymbol{\lambda}_{i}^{T} \mathbf{c}_{i}
$$

permits the necessary conditions to be written

$$
\nabla_{\mathbf{x}_{i}} \mathcal{L}=0, \quad i=1,2, \ldots, n, \quad \mathbf{c}\left(\mathbf{x}_{c}\right)=0 .
$$

The basic algorithmic approach involves the use of Newton's method or one of its variants to solve this nonlinear system. The resulting system determining corrections $\mathbf{d} \mathbf{x}_{c}$ and $\mathbf{d} \boldsymbol{\lambda}_{c}$ is

$$
\begin{aligned}
& \nabla_{\mathbf{x x}}^{2} \mathcal{L} \mathbf{d} \mathbf{x}_{c}+\nabla_{\mathbf{x} \lambda}^{2} \mathcal{L} \mathbf{d} \boldsymbol{\lambda}_{c}=-\nabla_{\mathbf{x}} \mathcal{L}^{T}, \\
& \nabla_{\mathbf{x}} \mathbf{c}\left(\mathbf{x}_{c}\right) \mathbf{d} \mathbf{x}_{c}=C \mathbf{d x}_{c}=-\mathbf{c}\left(\mathbf{x}_{c}\right),
\end{aligned}
$$

where the block bidiagonal matrix $C$ is defined in equation (13). The sparsity is a consequence of the trapezoidal rule. Here $\nabla_{x x}^{2} \mathcal{L}$ is block diagonal while $\nabla_{\mathrm{x} \lambda}^{2} \mathcal{L}=C^{T}$ is block bidiagonal. Nocedal and Wright [9] connect these equations to necessary conditions for the solution of a quadratic program. This leads to consideration of two main solution approaches.

Elimination The constraint equations (17) is solved for $\mathbf{d x}_{i}, i=2, \ldots, n-$ 1 , in terms of $\mathbf{d x}_{1}$ and $\mathbf{d x}_{n}$ as in equation (14). This permits the quadratic program to be reduced to a problem in just these variables with the constraint determined by the last row of the factored matrix. Second order sufficiency conditions must still be satisfied for this reduced problem. This is discussed by Li, Osborne and Prvan [8] and work they cited. This approach has been tested for boundary value stable problems. Simpler elimination schemes are possible, but these correspond essentially to simple shooting [1] and cannot be boundary value stable. 
Null space An alternative approach which does not depend on a boundary value formulation is based on the factorization

$$
C^{T}=\left[\begin{array}{ll}
Q_{1} & Q_{2}
\end{array}\right]\left[\begin{array}{l}
U \\
0
\end{array}\right] .
$$

Then the Newton equations are

$$
\left[\begin{array}{cc}
Q^{T} \nabla_{\mathbf{x x}}^{2} \mathcal{L} Q & {\left[\begin{array}{c}
U \\
0
\end{array}\right]} \\
{\left[\begin{array}{ll}
U^{T} & 0
\end{array}\right]} & 0
\end{array}\right]\left[\begin{array}{c}
Q^{T} \mathbf{d x}_{c} \\
\mathbf{d} \boldsymbol{\lambda}_{c}
\end{array}\right]=-\left[\begin{array}{c}
Q^{T} \nabla_{\mathbf{x}} \mathcal{L}^{T} \\
\mathbf{c}
\end{array}\right] .
$$

These are solved in the sequence

$$
\begin{aligned}
U^{T} Q_{1}^{T} \mathbf{d} \mathbf{x}_{c} & =-\mathbf{c} \\
Q_{2}^{T} \nabla_{\mathbf{x x}}^{2} \mathcal{L} Q_{2} Q_{2}^{T} \mathbf{d} \mathbf{x}_{c} & =-Q_{2}^{T} \nabla_{\mathbf{x x}}^{2} \mathcal{L} Q_{1} Q_{1}^{T} \mathbf{d} \mathbf{x}_{c}-Q_{2}^{T} \nabla_{\mathbf{x}} \mathcal{L}^{T} \\
U \mathbf{d} \boldsymbol{\lambda}_{c} & =-Q_{1}^{T} \nabla_{\mathbf{x x}}^{2} \mathcal{L} \mathbf{d} \mathbf{x}_{c}-Q_{1}^{T} \nabla_{\mathbf{x}} \mathcal{L}^{T}
\end{aligned}
$$

Sufficient conditions are just the second order sufficiency conditions

1. The matrix $C$ has full row rank so the linearized constraints are linearly independent.

2. The matrix $Q_{2}^{T} \nabla_{\mathrm{xx}}^{2} \mathcal{L} Q_{2}$ is nonsingular.

Remark 6 The null space method does not depend explicitly on techniques associated with boundary value problem solution methods. Thus it is of interest to ask if it possesses wider stability tolerances. Discussion of the method's properties is complicated by the presence of the Lagrange multipliers for which initial estimates have to be provided. Typically this is done by computing the generalized inverse solution to the necessary condition

$$
C^{T} \boldsymbol{\lambda}_{c}+\nabla_{\mathbf{x}} F^{T}=0
$$

at the initial point [9]. Note this equation has formal similarity to the discretization of the adjoint differential equation and so could connect the null space method back to stability questions. 
TABLE 4: Stability test: comparison of exact and computed values.

\begin{tabular}{ccc}
\multicolumn{4}{c}{ test results $n=11$} \\
.87665 & -.97130 & -1.0001 \\
.74089 & -1.0987 & -1.3432 \\
.47327 & -1.2149 & -1.6230 \\
.11498 & -1.3427 & -1.8611 \\
-.32987 & -1.4839 & -2.0366 \\
-.85368 & -1.6400 & -2.1250 \\
-1.4428 & -1.8125 & -2.1018 \\
-2.0773 & -2.0031 & -1.9444 \\
-2.7309 & -2.2137 & -1.6330 \\
-3.3719 & -2.4466 & -1.1526
\end{tabular}
particular integral $Q_{1}^{T} x$

$\begin{array}{rrr}.87660 & -.97134 & -1.0001 \\ .74083 & -1.0988 & -1.3432 \\ .47321 & -1.2150 & -1.6231 \\ .11491 & -1.3428 & -1.8612 \\ -.32994 & -1.4840 & -2.0367 \\ -.85376 & -1.6401 & -2.1250 \\ -1.4429 & -1.8125 & -2.1019 \\ -2.0774 & -2.0032 & -1.9444 \\ -2.7310 & -2.2138 & -1.6331 \\ -3.3720 & -2.4467 & -1.1527\end{array}$

Remark 7 If the null space method is applied to the Mattheij problem described in Example 1 with initial estimate $\mathbf{x}_{c}=0$ then the first step solves $C \mathbf{d x}_{c}=\mathbf{q}_{c}$. It follows that $Q_{1}^{T} \mathbf{d} \mathbf{x}_{c}=U^{-T} \mathbf{q}_{c}$ is independent of the boundary conditions and thus should estimate the corresponding term derived from the particular integral. That is

$$
U^{-T} \mathbf{q}_{c} \approx Q_{1}^{T} \operatorname{vec}\left\{\exp \left(t_{i}\right)\left[\begin{array}{l}
1 \\
1 \\
1
\end{array}\right]\right\} .
$$

Computed and exact results are displayed in Table 4 in the interesting case $h=0.1$. The results suggest that the null space method can exploit distability. 


\section{In conclusion}

It has been a deliberate intention to give the estimation problem more attention than it commonly receives [1, 6 , e.g.]. Even so, it has been the role of stability questions that has been emphasized. The estimation literature is mostly in the form of case studies. BVP formulations are often favoured. However, it is not always clear that the ODE is severely IVP unstable. It may be just that the BVP formulation has better convergence properties in the optimization problem. The estimation problem is a constrained optimization problem whether formulated explicitly (Simultaneous) or implicitly (Embedding). In neither context is it a completely conventional statistical estimation problem. This aspect is beginning to attract attention [13]. Some work remains to be done to fully prove the simultaneous method, but evidence of its effectiveness is accumulating. Recent advances include adapting collocation techniques to fit seamlessly within the simultaneous framework, and a large sample convergence rate result for the Bock iteration which involves a Gauss-Newton-like simplification of the Newton equations.

\section{References}

[1] U. M. Ascher, R. M. M. Mattheij, and R. D. Russell, Numerical solution of boundary value problems for ordinary differential equations, SIAM, Philadelphia, 1995. C906, C921, C924

[2] J. C. Butcher, Numerical methods for ordinary differential equations, John Wiley and Sons, 2003. C906

[3] G. Dahlquist, Convergence and stability in the numerical integration of ordinary differential equations, Math. Scand. 4 (1956), 33-53. C907, C916 
[4] G. Dahlquist, A special stability problem for linear multistep methods, BIT 3 (1963), 27-43. C905

[5] F. R. de Hoog and R. M. M. Mattheij, On dichotomy and well-conditioning in BVP, SIAM J. Numer. Anal. 24 (1987), 89-105. C908

[6] P. Deuflhard, Newton methods for nonlinear problems, Springer-Verlag, Berlin Heidelberg, 2004. C902, C924

[7] R. England and R. M. M. Mattheij, Boundary value problems and dichotomic stability, SIAM J. Numer. Anal. 25 (1988), 1037-1054. C909

[8] Z. Li, M. R. Osborne, and T. Prvan, Parameter estimation of ordinary differential equations, IMA J. Numer. Anal. 25 (2005), 264-285. C921

[9] J. Nocedal and S. J. Wright, Numerical optimization, Springer-Verlag, 1999. C917, C921, C922

[10] M. R. Osborne, On shooting methods for boundary value problems, J. Math. Analysis and Applic. 27 (1969), 417-433. C907

[11] M. R. Osborne, Cyclic reduction, dichotomy, and the estimation of differential equations, J. Comp. and Appl. Math. 86 (1997), 271-286. C918

[12] B. J. Quinn and E. J. Hannan, The estimation and tracking of frequency, Cambridge University Press, Cambridge, United Kingdom, 2001. C905

[13] J. O. Ramsay, G. Hooker, C. Cao, and C. Campbell, Estimating differential equations, preprint, Department of Psychology, McGill University, Montreal, Canada, 2005, p. 40. C924 


\section{Author address}

1. M. R. Osborne, Mathematical Sciences Institute, Australian National University, ACT 0200, Australia.

mailto:Mike.Osborne@maths. anu .edu.au 